\section{Los costos basados en actividades de Unidades Médico Quirúrgica y de Cuidados Medios en un hospital público}

\author{
LILIANA NERIZ ${ }^{1, \mathrm{a}}$, DIEGO CRUZ FERNÁNDEZ ${ }^{1, \mathrm{~b}}$, \\ DANITZA RODRÍGUEZ ARAYA ${ }^{1, \mathrm{c}}$, MIDORI SAWADA ${ }^{2}$
}

\section{Activity based costs of different units in a high complexity Chilean hospital}

Background: Hospitals must have management tools that allow them to fulfil all their requirements with the allocated resources. Aim: To evaluate the number of bed-days according to the discharge diagnosis in a High Complexity Hospital in Chile. Material and Methods: An activity Based Cost (ABC) calculation was used. The stages were gathering of procedures, identification of the resources used, analysis of discharge diagnosis, definition of cost objects, resource cost drivers, cost of procedures, procedures cost drivers and cost of the cost objects that corresponds to bed-day by each medical specialty. Costs were calculated for Medical-Surgical and Intermediate Care units. Results: There were significant differences in costs according to medical specialty when comparing the costs obtained using the Management information system (WinSig) and ABC. Respiratory, Renal and Circulatory-Cardiovascular specialties accounted for the most expensive bed-days. Conclusions: This study identified bed-day costs and provides disaggregated information about the causes of cost differences between medical specialties.

(Rev Med Chile 2020; 148: 17-29)

Key words: Costs and Cost Analysis; Health Care Costs; Hospital Costs.

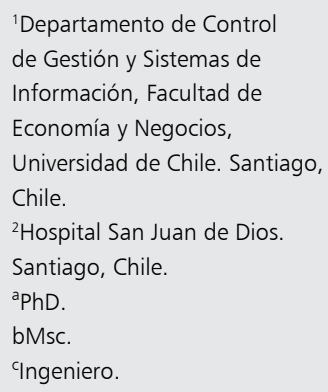

Financiación Proyecto Fondef IT13।10003.

Los autores declaran no tener conflictos e interés.

Recibido el 8 de agosto de 2019, aceptado el 2 de enero de 2020.

Correspondencia a:

Diego Cruz Fernández Msc Departamento de Control de Gestión y Sistemas de Información, Facultad de Economía y Negocios, Universidad de Chile. Santiago, Chile.

dcruz@fen.uchile.cl

\section{$\mathrm{L}$} a autogestión de los hospitales públicos requiere que estos cuenten con herramientas de gestión que les permitan mayor autonomía con respecto a los Servicios de Salud ${ }^{1}$. Así, se han introducido herramientasde gestión de costos como WINSIG-PERC y posteriormente los Grupos Relacionados por el Diagnóstico (GRD). Sin embargo, durante el año 2018 el déficit hospitalario alcanzó un record de $\$ 801.692$ millones $^{2}$, lo que corresponde a un alza de $27 \%$ con respecto al 2017.

En un Hospital de Alta Complejidad los pacientes generalmente ingresan por el Servicio de Urgencias o por interconsultas derivadas de los establecimientos de Atención Primaria, no todos los pacientes son resueltos en estas instancias ya que la indicación clínica puede requerir uso de cama hospitalaria. La principal vía de egresos de pacientes son los servicios de Hospitalización, por tanto es crítico para las instituciones gestionar el uso de camas y sus recursos involucrados, ya que éstos representan 58\% del gasto en los establecimientos terciarios ${ }^{3}$.

En el 2015 se facilita el acceso a camas del sector privado a través de mecanismos de pago por GRD, en el cual se paga un monto fijo convenido que cubre la resolución integral del paciente hasta su egreso dependiendo de su tipificación ${ }^{4}$. Sin embargo, sin un conocimiento efectivo de los costos surge la duda de cómo es posible identificar que el estado está pagando un precio razonable por estos servicios.

Para lograr una valorización de los costos de 
las prestaciones más detallada es necesario validar si el sistema de costeo del hospital lo permite, ya que la mayoría de éstos se basan en asignaciones volumétricas que no proporcionan la información adecuada para determinar de manera más precisa el costo de los servicios ${ }^{5,6}$. Una alternativa a estos sistemas es el Costeo Basado en Actividades $(\mathrm{ABC})$, el cual considera que los recursos de una organización son consumidos por distintas actividades y a su vez combinaciones de estas mismas deben ejecutarse para entregar un producto y/o servicio. Al costear actividades, es factible valorizar el objeto de costo (producto o servicio) con mayor exactitud y obtener información para evaluar la eficiencia de los distintos procesos, proporcionando una base más precisa para la toma de decisiones a nivel directivo ${ }^{7}$.

Se han realizado aplicaciones de $\mathrm{ABC}$ en servicios de hospitalización, algunas propuestas consideran calcular el costo de un "día cama estándar", ya que se reconoce la complejidad de formar paqueteslas actividades para cada paciente ${ }^{8}$. Otros casos han analizado los costos según el diagnóstico de los pacientes atendidos, lo que permite evidenciar diferencias significativas de costos entre especialidades ${ }^{9,10}$. Por otro lado, resultados de $\mathrm{ABC}$ exponen también diferencias con respecto a los aranceles fijos cobrados ${ }^{11}$, que muestran una posición desventajosa en términos financieros, si las tarifas no logran absorber la totalidad de los costos asociados para otorgar las prestaciones.

Este estudio tiene como objetivo valorizar el costo de día cama promedio según diagnóstico para Unidades de Hospitalización Médico Quirúrgico $(\mathrm{MQ})$ y de Cuidados Medios (CM) de un Establecimiento de Alta Complejidad en Chile. Se utiliza como base la metodología desarrollada en el Proyecto de Investigación FONDEF IT13I10003 "Una herramienta para la mejora de la gestión y valoración de prestaciones de hospitales públicos". La principal contribución de este estudio es que modela el comportamiento de unidades de hospitalización con tal nivel de detalle, que al aplicarse de manera constante, permite mejorar la gestión en esta y otras unidades similares.

\section{Material y Método}

Con el fin de simplificar la metodología aplicada del proyecto Fondef, se consideraron como actividades los procedimientos clínicos, ya que estos son una serie de pasos estandarizados que se realizan con el mismo orden lógico y consumen los mismos recursos. Para efectos de estudio se excluyen los gastos provenientes de unidades de apoyo clínico y de las actividades administrativas. Además los costos de los procedimientos clínicos asociados a la hospitalización según especialidad no consideran los costos directos de los tratamientos clínicos específicos.

Como instrumentos de medición se utilizó la observación directa de los procedimientos realizados y el criterio experto, que permitieron construir el diccionario de actividades, en este caso de procedimientos, y la estimación del consumo de los recursos involucrados. Los datos con los que se trabajó corresponden a egresos hospitalarios del año 2017. De un total de 7.514 egresos hospitalarios relativos a 521 diagnósticos, se consideran en el estudio 6.096 egresos, que constituyen los 111 diagnósticos más recurrentes, es decir, aquellos que ocurren al menos 12 veces al año. Los diagnósticos representan $80 \%$ de los días de estada correspondientes del período.

La Figura 1 muestra las etapas de esta metodología que son: levantamiento de procedimientos, identificación de recursos utilizados, análisis de diagnósticos de egreso, definición de objetos del costo, inductores de costo de recursos, costo de los procedimientos, inductores de procedimientos y valor del objeto de costo, que corresponde al día cama por especialidad.

A continuación se detallan cada una de las etapas.

\section{Levantamiento de procedimientos}

Consiste en un análisis de los procesos que se llevan a cabo en MQ y CM, de manera de obtener información para identificar los procedimientos requeridos para cada paciente dependiendo de su diagnóstico de egreso (DE).

\section{Identificación de recursos utilizados}

Una vez levantados los procedimientos se reconocen los recursos que se utilizan en cada uno de ellos, clasificados en insumos y medicamentos, que constituyen los gastos directos a los objetos del costo, y otros de naturaleza general, tales como: gastos en recursos humanos, (gastos de personal que realiza procedimientos clínicos), gastos generales (agua, energía, gas y arriendo de espacio físico), depreciación de activos fijos y equipamiento, 


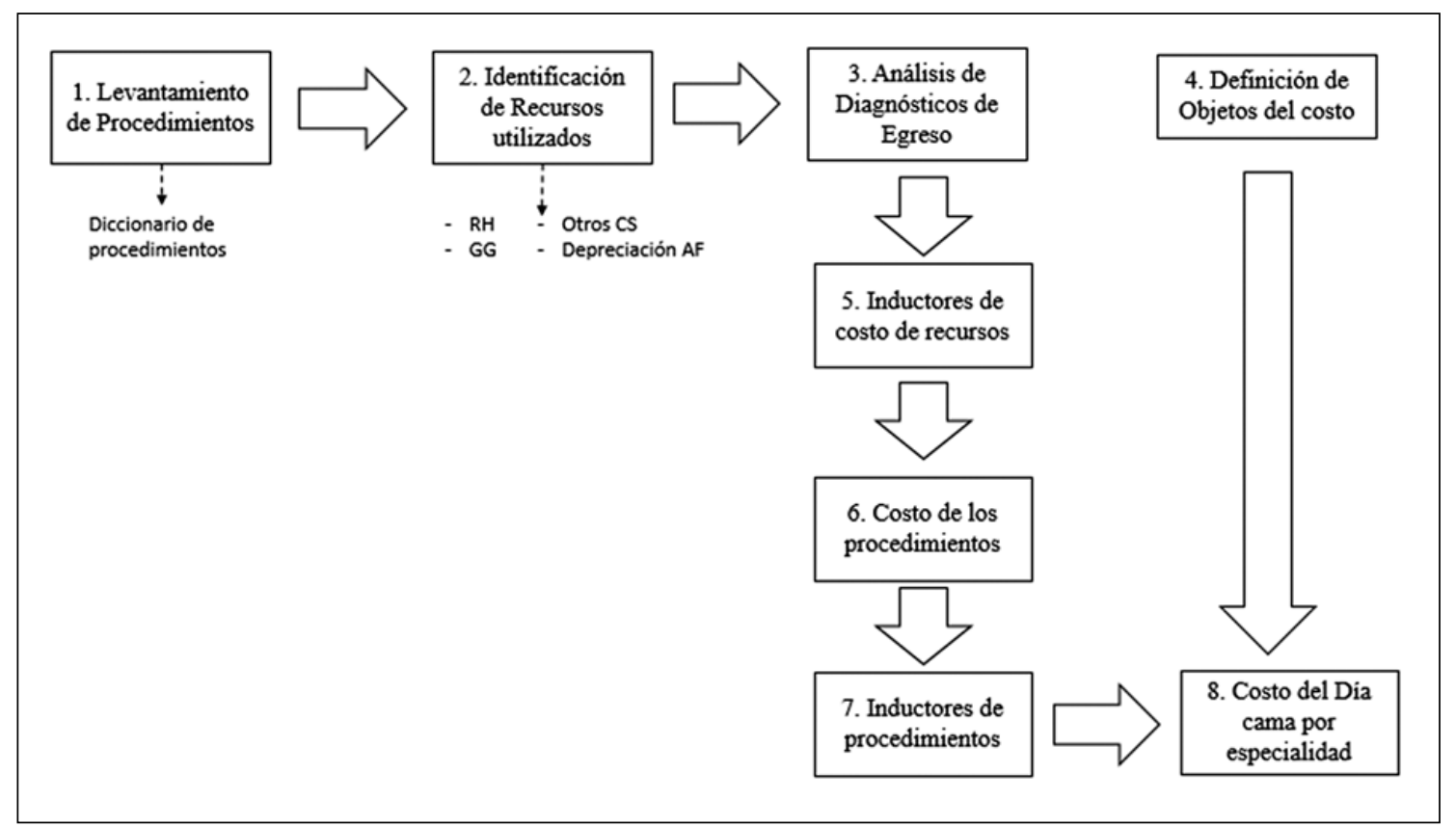

Figura 1. Metodología de Aplicación Sistema de Costeo ABC para unidades de Hospitalización (Elaboración propia, basado en Kaplan y Cooper).

y otros costos de servicio (gastos de oficina, ropa de cama, termómetros, etc.).

\section{Análisis de diagnósticos de egreso}

De los datos estadísticos del hospital se obtuvieron los diagnósticos de egreso, para MQ fueron 103 y para CM 43, considerando que hay 35 diagnósticos que son comunes para ambas unidades. La información disponible corresponde a los egresos anuales de pacientes y sus días de estada totales al año que fueron 42.217 para MQ y para CM 18.221.

\section{Definición de objeto de costo}

Se definen como objetos de costo los días cama según diagnóstico de egreso, agrupados por especialidad: respiratorio, digestivo, renal, ginecológico, traumatológico, neurológico y circulatorio-cardiovascular.

\section{Inductores de costos de recursos}

Corresponden a las bases de asignación que relacionan el uso de los recursos con los procedimientos levantados. Los inductores de cada tipo de recursos se ven representados en la Tabla 1.

Tabla 1. Inductores para cada tipo de recurso

\begin{tabular}{|lcc|}
\hline Tipo de Recursos & Inductor & \multicolumn{1}{c|}{ Métrica } \\
Recurso humano & Tiempo & $\begin{array}{r}\text { Total de minutos del recurso j para } \\
\text { la realización de procedimiento i }\end{array}$ \\
Gepros generales & Tiempo & $\begin{array}{c}\text { Total de minutos del recurso j para } \\
\text { la realización de todos los procedimientos }\end{array}$ \\
Otros costos de servicio & Tiempo & $\begin{array}{c}\text { Total de usos del recurso j para } \\
\text { la realización de procedimiento i }\end{array}$ \\
\cline { 2 - 2 } & Cuota de uso & Total de usos del recurso j para \\
\hline
\end{tabular}


Para obtener la información de dichos inductores fue necesario conocer en primera instancia el número total de cada procedimiento $i$ realizado al año, dado los egresos anuales de pacientes. La Figura 2 muestra la forma en que se calculó dicho valor.

En que:

$N^{\circ}$ Total proc $i$ : $\mathrm{N}^{\circ}$ total de procedimientos realizados al año para cada procedimiento $i$.

EgrePac: todos los pacientes que egresaron de cada unidad durante el año.

Cuotas de proc i por DE $z$ : $\mathrm{N}^{\circ}$ de veces que se realiza cada procedimiento $i$ en un día cama a un paciente con un diagnóstico de egreso $z$. En donde el valor de $z$ va desde 1 a 103 para MQ y de 1 a 43 para CM.

Días est: todos los días de estada que permanecieron todos los pacientes que egresaron en ambas unidades durante el año.

Con el $\mathrm{N}^{\circ}$ total de procedimientos realizados al año, se procede a calcular el valor del inductor para cada grupo de recursos. Para conocer el valor del inductor de los tres primeros grupos de recursos mencionados en la Tabla 1, primero se calcula el total de minutos que cada recurso participa en la realización de cada uno de los procedimientos, cuyo cálculo se ve reflejado en la Figura 3.

En que:

Total min rec $j$ total proc $i$ : $\mathrm{N}^{\circ}$ total de minutos en que un recurso $j$ participa en la realización del total de procedimientos $i$ al año, para cada procedimiento.

$N^{\circ}$ Total proc i: proveniente de ecuación 1.

$N^{\circ}$ min rec j para un proc $i$ : $\mathrm{N}^{\circ}$ de minutos en que participa el recurso $j$ en realizar un procedimiento $i$.

Para terminar de conocer el valor del inductor se necesita conocer el tiempo total que el recurso $j$ participa en la realización de todos los procedimientos, el cual se calcula para cada recurso como la sumatoria de los valores calculados en la ecuación 2 para todos los procedimientos. Para los tres primeros grupos de recursos el inductor queda construido de la forma indicada (Tabla 1).

Para conocer el valor del inductor del último grupo de recurso, otros costos de servicio, mencionado en la Tabla 1, se calculó el uso total de cada recurso para realizar cada uno de los procedimientos, según Figura 4.

En que:

Total usos rec $j$ total proc $i$ : Uso total de un recurso $j$ para realizar el total de procedimiento $i$ al año, para cada procedimiento.

$N^{\circ}$ Total proc i: proviene de la ecuación 1.

Cuota de uso rec j para un proc $i$ : $\mathrm{N}^{\circ}$ de usos del recurso $j$ para la realización de un procedimiento $i$, para cada procedimiento.

Dichas cuotas fueron levantadas mediante entrevistas y criterio experto.

Así, para terminar de calcular el valor del inductor se necesitan los usos totales del recurso $j$ para la realización de todos los procedimientos, el cual se calcula para cada recurso como la sumatoria de los valores calculados en la ecuación 3 para todos los procedimientos. De esta forma, quedan construidos los valores de los 4 tipos de inductores. En el anexo 1 se muestra un ejemplo del cálculo de las ecuaciones 1, 2 y 3.

\section{No total proc $i=$ Egre Pac $*$ cuotas de proc $i * D E z *$ dias est}

Figura 2. Ecuación 1 para calcular el número total de procedimientos.

Total min rec $j$ total proc $i=N^{\circ}$ total proc $i * N^{\circ}$ min rec $j$ para un proc $i$

Figura 3. Ecuación 2 para calcular el número total de minutos que se utilizan para la realización de cada procedimiento.

Total usos rec $j$ total proc $i=N^{0}$ total proc $i *$ cuota de uso rec $j$ para un proc $i$

Figura 4. Ecuación 3 para calcular el número total de usos que se utilizan para la realización de cada procedimiento. 
Para realizar la asignación de los costos de los recursos a los procedimientos se multiplicó el valor de los inductores calculados anteriormente por los montos de gastos anuales de cada tipo de recurso. El origen de cada uno de dichos valores se observa en la Tabla 2.

\section{Costo de los procedimientos}

En esta etapa se calcula el costo anual indirecto de cada procedimiento que viene dado por el consumo de recursos asignado a cada procedimiento dadoel egreso de pacientes.

\section{Inductores de los procedimientos}

Se definen los inductores de costos de los procedimientos para la asignación de sus costos a los objetos de costos, en este caso días cama por especialidad. Los inductores de cada procedimiento se ven representados en la Tabla 3 para MQ y la Tabla 4 para CM.

Para obtener la información de dichos inductores fue necesario identificar cuántos procedimientos se realizaban al año para cada día cama por especialidad (DCE). Dicha información se obtuvo del cálculo de la Figura 5.

Tabla 2. Costos de los recursos

\begin{tabular}{|ll|}
\hline Tipo de recursos & Costo asignado \\
\hline Recurso humano indirecto & Costo anual de los recursos humanos de las unidades \\
\hline Gastos generales & Costo anual del hospital prorrateado a las unidades por $\mathrm{m}^{2}$ utilizados \\
\hline Activos fijos & Costo anual de las depreciaciones de los activos de las unidades \\
\hline Otros costos de servicio & Costo anual de los otros costos de servicio de las unidades \\
\hline
\end{tabular}

Tabla 3. Inductores de procedimiento y costos anuales indirectos de procedimientos Unidad Médico Quirúrgico

\begin{tabular}{|c|c|c|}
\hline \multicolumn{3}{|c|}{ UMQ } \\
\hline Procedimientos & Inductor de Procedimiento & Cl Anual \\
\hline Alta paciente & $\mathrm{N}^{\circ}$ de pacientes dados de alta & $\$ 888.718 .045$ \\
\hline Ingreso paciente & $\mathrm{N}^{\circ}$ de pacientes ingresados & $\$ 551.200 .694$ \\
\hline Administración de medicamentos & $\mathrm{N}^{\circ}$ de administraciones de medicamentos realizadas & $\$ 400.941 .477$ \\
\hline Alimentación paciente & $\mathrm{N}^{\circ}$ de veces que se alimenta al paciente & $\$ 318.365 .741$ \\
\hline Aseo paciente & $\mathrm{N}^{\circ}$ de pacientes aseados & $\$ 259.880 .018$ \\
\hline Control de signos vitales & $\mathrm{N}^{\circ}$ de controles de $\mathrm{SV}$ realizados & $\$ 94.495 .459$ \\
\hline Instalación catéter venoso periférico & $\mathrm{N}^{\circ}$ de instalaciones realizadas & $\$ 62.020 .583$ \\
\hline Toma de examen de sangre & $\mathrm{N}^{\circ}$ de exámenes realizados & $\$ 58.693 .064$ \\
\hline Preparación de flebos & $\mathrm{N}^{\circ}$ de flebos preparados & $\$ 31.135 .040$ \\
\hline Oxigenoterapia & $\mathrm{N}^{\circ}$ de oxigenoterapias realizadas & $\$ \quad 22.211 .990$ \\
\hline Curaciones & $\mathrm{N}^{\circ}$ de curaciones realizadas & $\$ 21.003 .679$ \\
\hline Instalación sonda foley & $\mathrm{N}^{\circ}$ de instalaciones realizadas & $\$ \quad 15.389 .150$ \\
\hline Electro cardiograma & $\mathrm{N}^{\circ}$ de electros realizados & $\$ \quad 10.255 .509$ \\
\hline Mantención de sonda foley & $\mathrm{N}^{\circ}$ de mantenciones realizadas & 7.348 .648 \\
\hline Retiro de sonda foley & $\mathrm{N}^{\circ}$ de retiros realizados & 6.802 .789 \\
\hline Instalación de sonda nasogástrica & $\mathrm{N}^{\circ}$ de instalaciones realizadas & 4.243 .133 \\
\hline
\end{tabular}


Tabla 4. Inductores de procedimiento y costos anuales indirectos de procedimientos Unidad Cuidados Medios

\begin{tabular}{|c|c|c|c|}
\hline \multicolumn{4}{|c|}{ UCM } \\
\hline Procedimientos & Inductor de procedimiento & & Cl Anual \\
\hline Alta paciente & $\mathrm{N}^{\circ}$ de pacientes dados de alta & $\$$ & 784.042 .579 \\
\hline Administración de medicamentos & $\mathrm{N}^{\circ}$ de administraciones de medicamentos realizadas & $\$$ & 531.840 .754 \\
\hline Alimentación al paciente & $\mathrm{N}^{\circ}$ de veces que se alimenta al paciente & $\$$ & 293.395 .562 \\
\hline Egreso o traslado de unidad & $\mathrm{N}^{\circ}$ de egresos o traslados & $\$$ & 223.177 .168 \\
\hline Instalación o retiro de catéter venoso central & $\mathrm{N}^{\circ}$ de instalaciones o retiros realizados & $\$$ & 181.115 .640 \\
\hline Procedimiento kinesiológico & $\mathrm{N}^{\circ}$ de procedimientos realizados & $\$$ & 164.986 .288 \\
\hline Ingreso paciente & $\mathrm{N}^{\circ}$ de ingresos realizados & $\$$ & 161.342 .534 \\
\hline Aseo paciente & $\mathrm{N}^{\circ}$ de pacientes aseados & $\$$ & 160.863 .825 \\
\hline Toma de examen de sangre & $\mathrm{N}^{\circ}$ de exámenes realizados & $\$$ & 86.962 .707 \\
\hline Instalación catéter venoso periférico & $\mathrm{N}^{\circ}$ de instalaciones realizadas & $\$$ & 67.519 .141 \\
\hline Instalación sonda foley & $\mathrm{N}^{\circ}$ de instalaciones realizadas & $\$$ & 50.821 .767 \\
\hline Curaciones & $\mathrm{N}^{\circ}$ de curaciones realizadas & $\$$ & 44.899 .744 \\
\hline Electro cardiograma & $\mathrm{N}^{\circ}$ de electros realizados & $\$$ & 31.632 .508 \\
\hline Mantención de sonda foley & $\mathrm{N}^{\circ}$ de mantenciones realizadas & $\$$ & 14.457.181 \\
\hline Retiro de sonda foley & $\mathrm{N}^{\circ}$ de retiros realizados & $\$$ & 12.854 .485 \\
\hline Realización de diálisis & $\mathrm{N}^{\circ}$ de diálisis realizadas & $\$$ & 11.897 .218 \\
\hline
\end{tabular}

\section{$N$ o total de proc para cada DCE = Cuotas de proc $* D C E *$ dias est $* D C E$}

Figura 5. Ecuación 4 para calcular el número total de procedimientos para día cama por especialidad.

\section{Total de procedimientos i realizados para el DCE $j$ Total de precedimientos i realizados para todos los DCE}

Figura 6. Ecuación 5 para construir el inductor de procedimientos.

En que:

No total de proc para cada DCE: $\mathrm{N}^{\circ}$ total de procedimientos $i$ realizados para cada DCE durante el año.

Cuotas de proc por DCE: $\mathrm{N}^{\circ}$ de veces que deben realizarse los procedimientos a un paciente en un día cama dependiendo de su diagnóstico. Dichas cuotas fueron levantadas mediante entrevistas y criterio experto.

Días estada por DCE: total de días de estada que permanecieron todos los pacientes que egresaron de las unidades con determinado DCE durante el año.

Para construir la base de asignación del costo fue necesario conocer el total de procedimientos $i$ realizados para todos los días cama por especialidad, el que se calculó como la sumatoria por procedimiento de la ecuación 4 para todos los DCE. Así, la base de asignación viene dada por la Figura 6. 
De esta forma, la asignación del costo se efectuó multiplicando las bases de asignación por el costo anual indirecto de cada procedimiento.

\section{Valor del objeto de costo}

En esta etapa se obtiene el costo anual indirecto para cada especialidad, al cual se suma el costo anual directo, que corresponde a las canastas de insumos y medicamentos utilizadas para cada tipo de DE por especialidad. Finalmente, la suma de estos costos directos e indirectos se dividen por los días de estada anuales para cada especialidad y se obtiene así, el costo de un día cama promedio para cada tipo de especialidad.

\section{Resultados}

Utilizando esta metodología, los costos indirectos anuales de los procedimientos que se obtuvieron tanto para MQ como para CM pueden verse en la Tabla 3 y en la Tabla 4 respectivamente.

El total de procedimientos realizados al año para cada especialidad se obtuvo mediante la ecuación 4 para cada DCE, cuyos valores se observan en la Tabla 5 para MQ y en la Tabla 6 para CM.

Las Tablas 7 y 8 muestran, tanto para MQ como para CM, cómo se distribuyeron los costos indirectos de cada procedimiento a los objetos de costo finales que son los DCE para cada

Tabla 5. Total de procedimientos anuales realizados por especialidad en Unidad Médico Quirúrgico

\begin{tabular}{|c|c|c|c|c|c|c|c|c|c|}
\hline UMQ & $\begin{array}{l}\text { Respira- } \\
\text { torio }\end{array}$ & Renal & $\begin{array}{l}\text { Circulatorio- } \\
\text { Cardiovascular }\end{array}$ & $\begin{array}{l}\text { Gineco- } \\
\text { logía }\end{array}$ & $\begin{array}{l}\text { Trauma- } \\
\text { tológico }\end{array}$ & $\begin{array}{l}\text { Diges- } \\
\text { tivo }\end{array}$ & Otros & $\begin{array}{l}\text { Neuro- } \\
\text { lógico }\end{array}$ & Total \\
\hline $\begin{array}{l}\text { Administración de } \\
\text { medicamentos }\end{array}$ & 20.649 & 4.977 & 20.769 & 4.849 & 19.020 & 39.479 & 13.470 & - & 123.213 \\
\hline Alta paciente & 6.301 & 1.659 & 6.523 & 1.564 & 4.755 & 14.208 & 6.970 & 237 & 42.217 \\
\hline Aseo paciente & 13.875 & 4.977 & 16.495 & 4.378 & 12.141 & 28.784 & 13.292 & 237 & 94.179 \\
\hline $\begin{array}{l}\text { Control de signos } \\
\text { vitales }\end{array}$ & 18.327 & 4.977 & 19.062 & 4.692 & 14.265 & 39.716 & 13.152 & - & 114.191 \\
\hline Curaciones & - & - & 233 & 157 & 4.097 & 5.414 & 908 & - & 10.809 \\
\hline $\begin{array}{l}\text { Instalación catéter } \\
\text { venoso periférico }\end{array}$ & 6.109 & 1.659 & 6.354 & 1.564 & 4.289 & 11.130 & 4.069 & - & 35.174 \\
\hline Electrocardiograma & 654 & - & 4.333 & - & - & - & 379 & - & 5.366 \\
\hline $\begin{array}{l}\text { Preparación de } \\
\text { flebos }\end{array}$ & 2.764 & 2.876 & 4.853 & 157 & 7.566 & 4.688 & 1.094 & - & 23.998 \\
\hline Ingreso paciente & 6.301 & 1.659 & 6.523 & 1.564 & 4.755 & 14.208 & 6.970 & 237 & 42.217 \\
\hline Oxigenoterapia & 12.669 & - & 4.011 & - & - & 493 & 1.038 & - & 18.211 \\
\hline $\begin{array}{l}\text { Instalación de } \\
\text { sonda nasogástrica }\end{array}$ & 685 & - & 210 & - & - & - & - & - & 895 \\
\hline $\begin{array}{l}\text { Instalación sonda } \\
\text { foley }\end{array}$ & 235 & 1.659 & 1.735 & 1.407 & 120 & 749 & 880 & - & 6.785 \\
\hline $\begin{array}{l}\text { Mantención de } \\
\text { sonda foley }\end{array}$ & 235 & 1.659 & 1.735 & 1.407 & 120 & 749 & 880 & - & 6.785 \\
\hline $\begin{array}{l}\text { Retiro de sonda } \\
\text { foley }\end{array}$ & 235 & 1.157 & 1.735 & 1.407 & 120 & 749 & 880 & - & 6.283 \\
\hline $\begin{array}{l}\text { Toma de examen } \\
\text { de sangre }\end{array}$ & 6.109 & 1.659 & 6.354 & 1.564 & 4.755 & 13.304 & 4.384 & - & 38.129 \\
\hline $\begin{array}{l}\text { Alimentación } \\
\text { paciente }\end{array}$ & 18.903 & 4.977 & 19.569 & 4.692 & 14.265 & 42.624 & 20.910 & 711 & 126.651 \\
\hline
\end{tabular}


Tabla 6. Total de procedimientos anuales realizados por especialidad en Unidad Cuidados Medios

\begin{tabular}{|c|c|c|c|c|c|c|c|c|}
\hline UCM & $\begin{array}{l}\text { Diges- } \\
\text { tivo }\end{array}$ & $\begin{array}{l}\text { Respi- } \\
\text { ratorio }\end{array}$ & Otros & $\begin{array}{l}\text { Circulatorio- } \\
\text { Cardiovascular }\end{array}$ & Renal & $\begin{array}{l}\text { Gineco- } \\
\text { logía }\end{array}$ & $\begin{array}{l}\text { Trauma- } \\
\text { tológico }\end{array}$ & Total \\
\hline $\begin{array}{l}\text { Administración de } \\
\text { medicamentos }\end{array}$ & 4.633 & 15.211 & 3.459 & 30.318 & 1.770 & 1.227 & 1.228 & 57.846 \\
\hline Alta paciente & 1.363 & 4.762 & 1.432 & 9.358 & 590 & 409 & 307 & 18.221 \\
\hline Aseo paciente & 3.757 & 14.286 & 4.178 & 27.746 & 1.770 & 1.227 & 921 & 53.885 \\
\hline Curaciones & 1.506 & 999 & 795 & - & 1.770 & 1.227 & 921 & 7.218 \\
\hline $\begin{array}{l}\text { Instalación o retiro } \\
\text { de catéter venoso } \\
\text { central }\end{array}$ & 497 & 2.738 & 314 & 6.950 & 1.087 & - & - & 11.586 \\
\hline $\begin{array}{l}\text { Instalación catéter } \\
\text { venoso periférico }\end{array}$ & 999 & 4.762 & 897 & 9.358 & 590 & - & 307 & 16.913 \\
\hline $\begin{array}{l}\text { Realización de } \\
\text { diálisis }\end{array}$ & - & - & - & - & 590 & - & - & 590 \\
\hline Electrocardiograma & 166 & 673 & 611 & 8.138 & - & - & - & 9.588 \\
\hline Ingreso paciente & 1.363 & 4.762 & 1.432 & 9.358 & 590 & 409 & 307 & 18.221 \\
\hline $\begin{array}{l}\text { Procedimiento } \\
\text { kinesiológico }\end{array}$ & 532 & 4.800 & 652 & - & - & 1.227 & 615 & 7.826 \\
\hline $\begin{array}{l}\text { Toma de examen } \\
\text { de sangre }\end{array}$ & 1.197 & 4.762 & 1.303 & 9.358 & 590 & 409 & 307 & 17.926 \\
\hline $\begin{array}{l}\text { Instalación sonda } \\
\text { foley }\end{array}$ & 166 & 333 & - & 2.847 & 590 & 409 & 153 & 4.498 \\
\hline $\begin{array}{l}\text { Mantención de } \\
\text { sonda foley }\end{array}$ & 166 & 333 & - & 2.847 & 590 & 409 & 153 & 4.498 \\
\hline $\begin{array}{l}\text { Retiro de sonda } \\
\text { foley }\end{array}$ & 166 & 333 & - & 2.847 & 497 & 409 & 153 & 4.405 \\
\hline $\begin{array}{l}\text { Egreso o traslado } \\
\text { de unidad }\end{array}$ & 1.363 & 4.762 & 1.432 & 9.358 & 590 & 409 & 307 & 18.221 \\
\hline $\begin{array}{l}\text { Alimentación al } \\
\text { paciente }\end{array}$ & 4.089 & 14.286 & 4.296 & 28.074 & 1.770 & 1.227 & 921 & 54.663 \\
\hline
\end{tabular}

unidad. Los valores observados se obtuvieron multiplicando las respectivas ecuaciones 5 para cada DCE por el costo total indirecto de cada procedimiento.

Las Tablas 9 y 10 muestran los datos anuales tanto de costos indirectos, como de los costos directos, considerando sus respectivos egresos totales, el total de días de estada y el costo total unitario promedio para cada DCE, en ambas unidades.

\section{Discusión y Conclusiones}

La metodología y los resultados obtenidos en este estudio presentan ciertas similitudes con trabajos previos $^{810}$ a nivel nacional, en cuanto se han enfocado en el costeo de especialidades o patologías. Sin embargo, uno de ellos ${ }^{10}$, solo lo aplica a UCI, unidad que no fue abordada en esta investigación, y el otro artículo $^{8}$ no presenta resultados. 


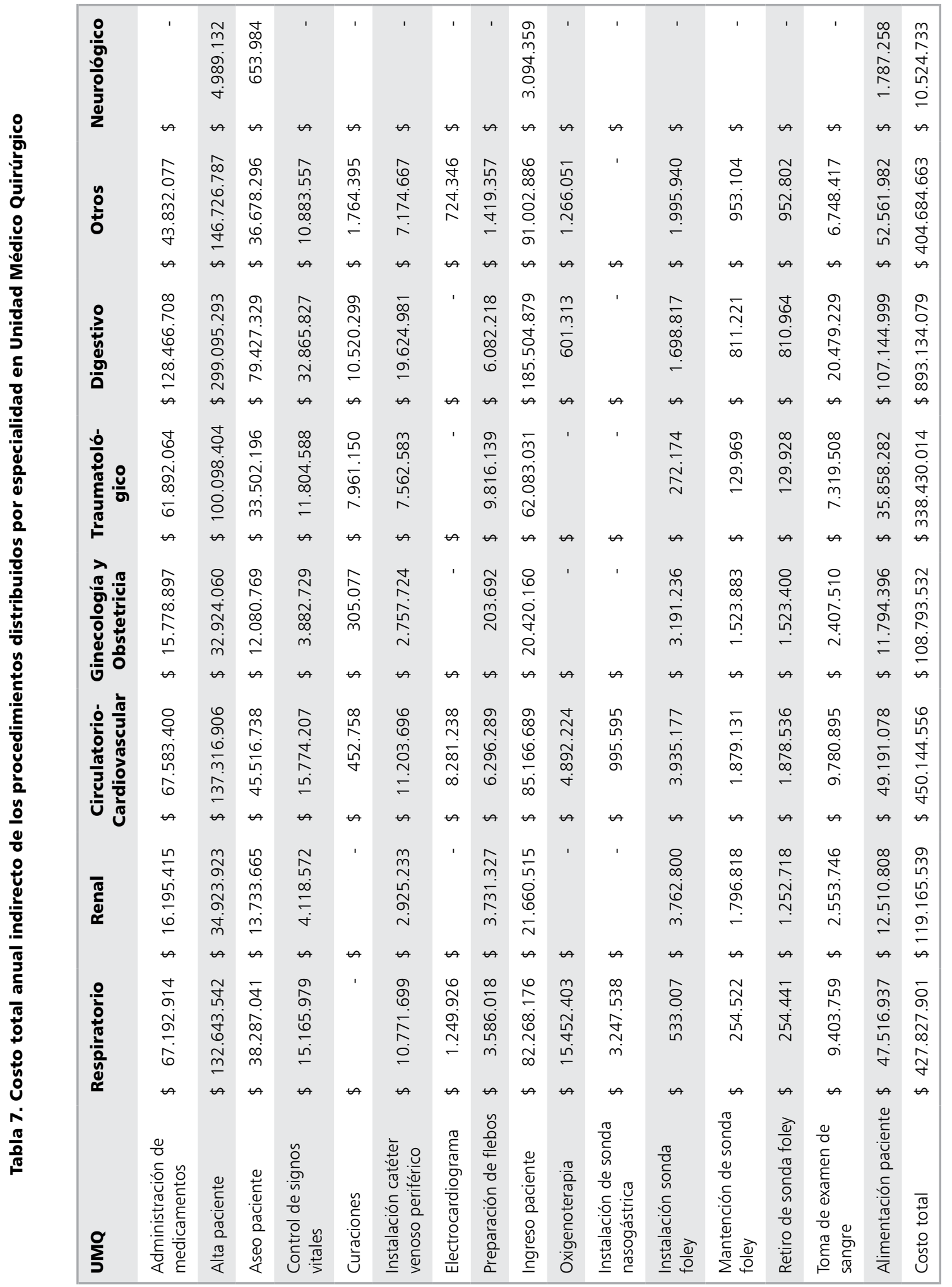




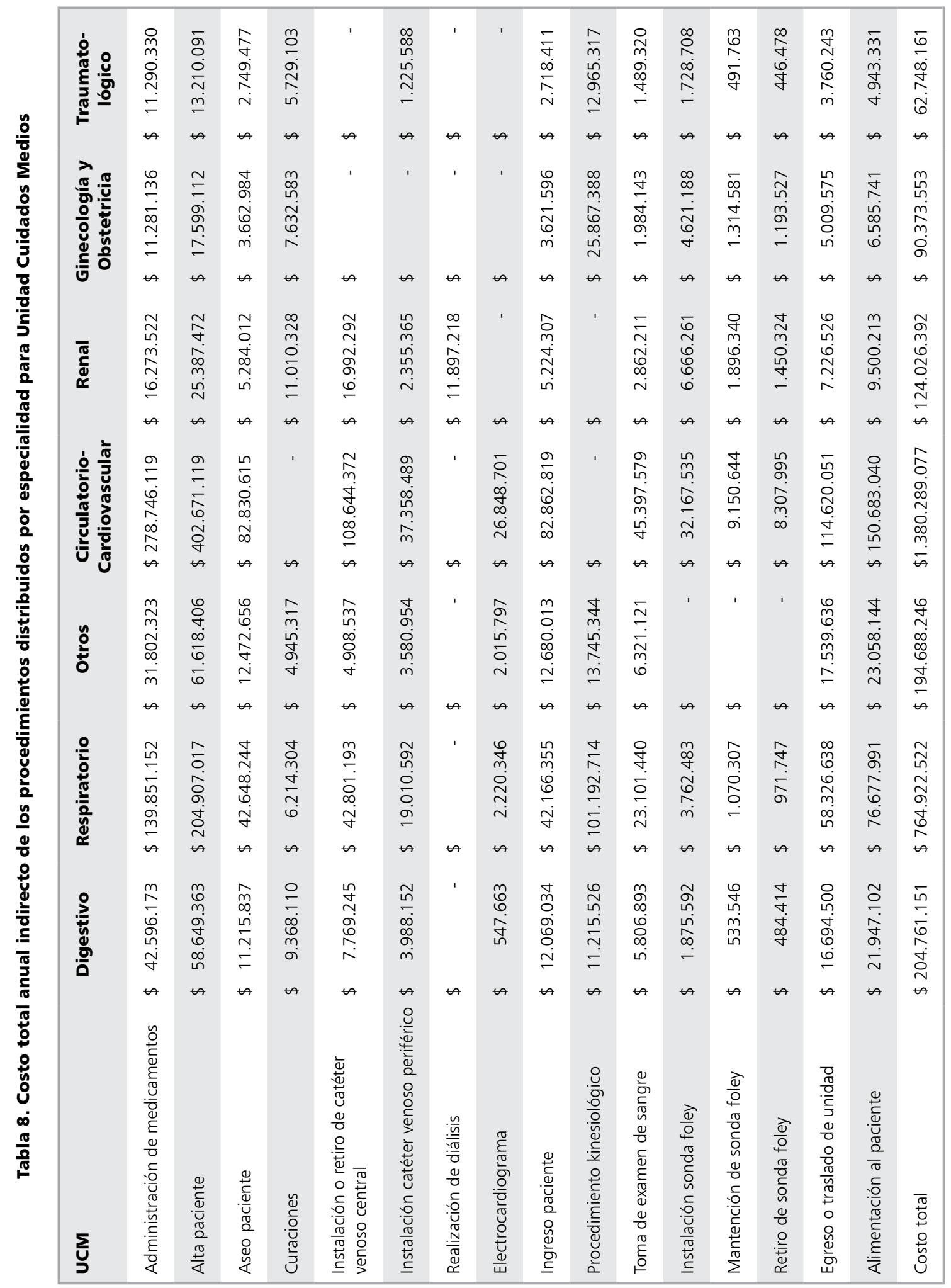



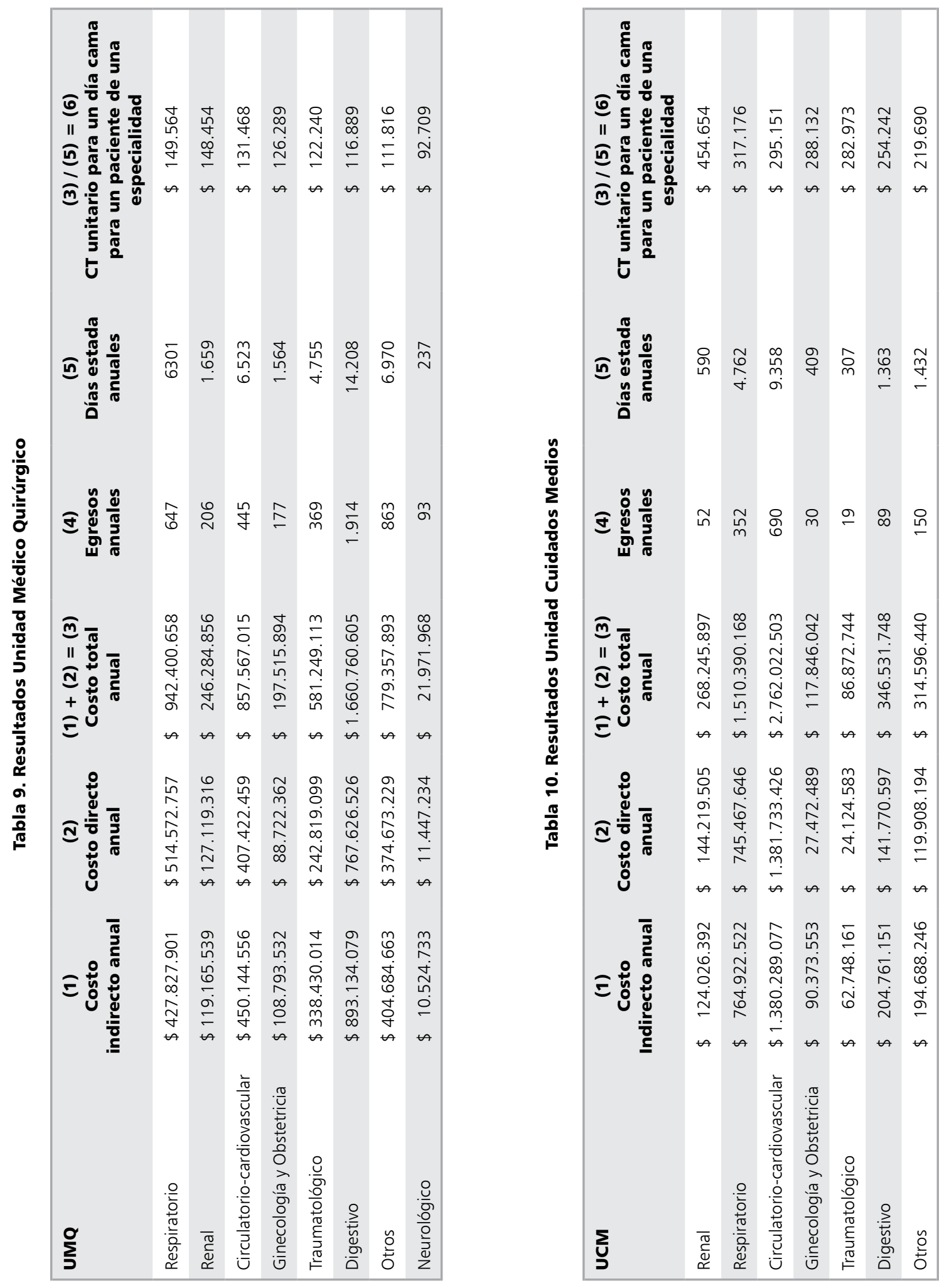

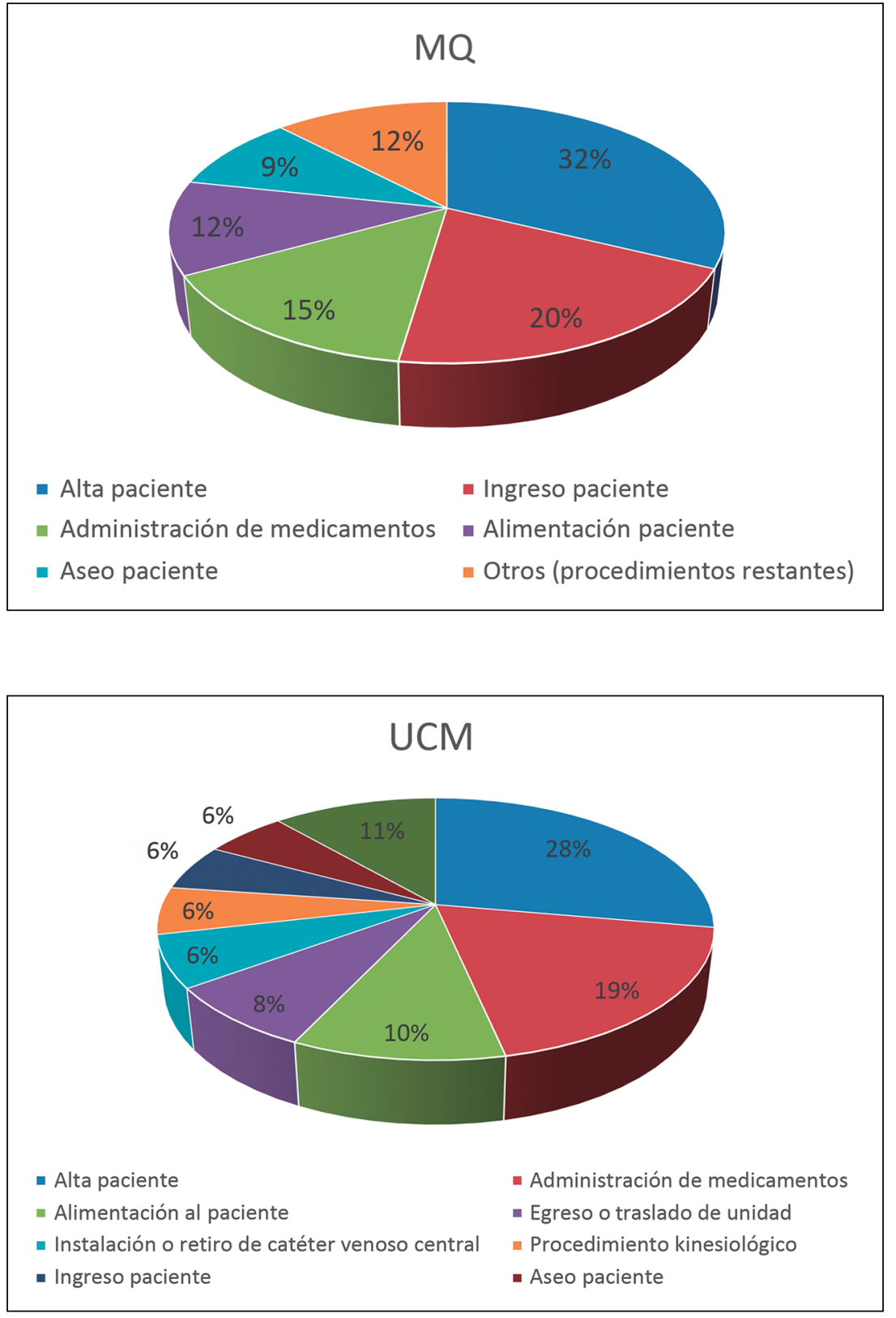

Figura 7. Distribución costos indirectos anuales distribuidos por procedimiento (Fuente: Tabla 3).
Figura 8. Distribución costos indirectos anuales distribuidos por procedimiento (Fuente: Tabla 4).
Este estudio permite identificar aquellos procesos o servicios más caros dentro de las unidades de hospitalización y entregar información detallada respecto de por qué algunas unidades consumen más recursos que otras. La Figura 7 muestra que el procedimiento con mayor costo indirecto anual para su realización, fue el Alta Paciente para MQ (32\% del total anual). Así mismo la Figura 8 muestra similar resultado para CM (28\% del total anual). Para ambas unidades, la mayor proporción de estos costos viene dado por el alto consumo de recursos humanos que utiliza este procedimiento.

Los DE que tuvieron un costo de día cama más alto fueron los Respiratorios y Renales para ambas unidades, según Tablas 9 y 10. Dichos valores vienen dados, para MQ, por los altos costos 
de los procedimientos de Alta Paciente, Ingreso Paciente y Administración de Medicamentos (Tabla 7). Para CM los altos costos se explican por los procedimientos de Alta Paciente, Administración de Medicamentos y Alimentación de Paciente (Tabla 8).

Los costos promedio de día de estada anual según metodología WinSIG para el 2017 fueron de \$127.404. En CM los días cama de todas las especialidades están subvaloradas con respecto al WinSIG, alcanzando hasta 250\% de diferencia como máximo, y en MQ hasta $17 \%$.

Los resultados de este estudio difieren de los del WinSIG principalmente porque este último no se diferencia el nivel de complejidad entre unidades (CM y MQ) y diagnósticos de egreso. Si bien es cierto al complementar los resultados del WinSIG con GRD se puede minimizar este sesgo, este reporte no entrega información detallada de la composición del costo asociada a cada prestación. El peso medio de un GRD y el precio base por sí solos no explican si los recursos están siendo asignados de forma más exacta.

\section{Referencias}

1. Artaza O. Los desafíos de la Autogestión Hospitalaria. Rev Chil Pediatri 2008; 79 (2): 127-30.

2. Sesión Comisión de Salud Cámara de Diputados "Estado de la deuda hospitalaria en el año 2018, sus efectos y medidas para enfrentarla durante el año 2019", celebrada el 23 de enero de 2019 en Congreso de Valparaíso. Disponible en: https://www.latercera.com/nacional/ noticia/deficit-hospitales-marco-nuevo-record-supero-los-801-mil-millones-2018/545889/ [Consultado en junio de 2019].
3. Ministerio de Salud. Distribución del gasto de salud por enfermedad, edad y sexo a nivel terciario de atención en Chile, año 2014. Disponible en https:// www.researchgate.net/profile/Cristobal_Cuadrado/ publication/328582489_Distribucion_del_gasto_de_salud_por_enfermedad_edad_y_sexo_a_nivel_terciario_de_atencion_en_Chile_ano_2014/links/5bd73eaa92851c6b2797266f/Distribucion-del-gasto-de-saludpor-enfermedad-edad-y-sexo-a-nivel-terciario-deatencion-en-Chile-ano-2014.pdf [Consultado en junio de 2019].

4. Pérez P. Informe Unidad de Gestión Centralizada de Camas. Período enero 2014-diciembre 2017. Disponible en https://www.minsal.cl/wp-content/uploads/2018/03/ Informe-UGCC-2014-2018.pdf [Consultado en junio de 2019].

5. Ross T. Analyzing Health Care Operations Using ABC. Journal of Health Care Finance 2004; 30 (3): 1-20.

6. Yereli A. Activity-Based Costing and Its Application in a Turkish University Hospital.Aorn Journal March 2009 Vol 89.

7. Baker J. Activity Based Costing and Activity based Management for Health Care. 1998 (p27).

8. Gallegos C, Medina A, Leyton C. Guía metodológica para determinar día cama mediante costeo ABC. Revista Médica de Risaralda 2015.

9. Popesko B, Novak P, Svetlik M. Applying the activity-based costing method to calculate the cost of hospitalization in the ent. TTEM, 317.

10. Alvear S, Canteros J, Jara J, Rodríguez, P. Costos reales de tratamientos intensivos por paciente y día cama. Rev Med Chile 2013; 141: 202-8.

11. Rajabi A. The role of activity based costing (ABC) system in governmental hospital services in Iran. Iranian Red Crescent Medical Journal 2008; 10 (2): 89-94. http://citeseerx.ist.psu.edu/viewdoc/download?doi=10.1.1.816.6578\&rep=rep1\&type $=$ pdf. 